\title{
Effect of vitamin D on the learning and memory ability of FGR rat and NMDA receptor expression in hippocampus
}

\author{
LU ZONG ${ }^{1 *}$, PING CHU ${ }^{2 *}$, PU HUANG ${ }^{1}$, YULIN GUO ${ }^{3}$ and $\mathrm{YE} \mathrm{LV}^{4}$ \\ ${ }^{1}$ Department of Gynaecology and Obstetrics, The First Affiliated Hospital of Xi'an Jiaotong University, Xi'an, \\ Shaanxi 710061; ${ }^{2}$ Department of Gynaecology and Obstetrics, Yantai Affiliated Hospital of Binzhou Medical University, \\ Yantai, Shandong 264100; ${ }^{3}$ Department of Gynaecology and Obstetrics, The Second Hospital of Yulin, \\ Yulin, Shaanxi 719000; ${ }^{4}$ Department of Behavioral Pediatrics, The Second Affiliated \\ Hospital of Xi'an Jiaotong University, Xi'an, Shaanxi 710004, P.R. China
}

Received December 22, 2016; Accepted April 28, 2017

DOI: $10.3892 / \mathrm{etm} .2017 .4523$

\begin{abstract}
The aim of this study is to investigate the effect of vitamin $\mathrm{D}(\mathrm{VD})$ on the learning and memory ability of fetal growth restriction (FGR) rat and the expression of NMDA receptor in hippocampus. The FGR models of rat were established through passive smoking, and divided into two groups randomly, i.e. the control group and the observation group. Rats were fed regular diet with the addition of VD in the observation group. The serum level of $25(\mathrm{OH})-\mathrm{D}_{3}$ was assayed in both groups of the rats at different time points using ELISA, and the learning and memory ability of rat aged 30 days were evaluated using Morris water maze and passive avoidance test. In addition, we also compared the brain weight of rats at different age and detected the mRNA expression of NRI subunit of NMDA receptor of hippocampus of rats aged 30 days using quantitative RT-PCR. After 7 days, the serum level of $25(\mathrm{OH}) \mathrm{D}_{3}$ in rats of the observation group was significantly higher than that of the control group $(\mathrm{P}<0.05)$; during the Morris water maze, we found no significant difference in comparison of the latency between the two groups in the first 2 days $(\mathrm{P}>0.05)$, but from the 3rd day, the latency of the observation group was shorter than that in the control group $(\mathrm{P}<0.05)$; in the passive avoidance test, no significant difference was identified when
\end{abstract}

Correspondence to: Dr Yulin Guo, Department of Gynaecology and Obstetrics, The Second Hospital of Yulin, 206 South Street, Yulin, Shaanxi 719000, P.R. China

E-mail: eo0022022@163.com

Dr Ye Lv, Department of Behavioral Pediatrics, The Second Affiliated Hospital of Xi'an Jiaotong University, 157 Xiwulu, Xi'an, Shaanxi 710004, P.R. China

E-mail: 1vye698@163.com

*Contributed equally

Key words: vitamin D, FGR rat, learning and memory ability, NMDA receptor in hippocampus comparing the electric shock times between the two groups in the first 2 days, but from the 3rd day, the electric shock times in the observation group were significantly lower than those in the control group $(\mathrm{P}<0.05)$; the brain weight of rats in the observation group on the 1st, 7th and 14th day were all lower than those in the control group $(\mathrm{P}<0.05)$, but the comparison of brain weight at 21st and 30th day showed no significant difference $(P>0.05)$ between the two groups. We also found that the mRNA and protein expression of NRI subunit of NMDA receptor in hippocampus was significantly higher in the observation group than in the control group $(\mathrm{P}<0.05)$. VD can increase the learning and memory ability of FGR rats, significantly ameliorating the cognitive dysfunction of FGR rat and improving the learning and memory ability of rats, which may be related to the upregulation of NRI subunit of NMDA receptor.

\section{Introduction}

Fetal growth restriction (FGR), also known as the intrauterine growth retardation, is a common kind of obstetrical complications. In clinical practice, FGR is diagnosed according to fetal weight which is two standard deviations less than the average of fetal weight at the same gestation age (1). FGR usually causes various diseases of newborns, such as asphyxia neonatorum, intrauterine fetal distress, meconium aspiration, hypothermia or even death. The incidence rate of FGR has reached as high as $15 \%$, and the mortality rate is almost 5 times as high as that in the healthy neonates (2). According to the relevant literature, the FGR in pregnancy induced by various factors can affect the regular development of fetal brain, and it can also damage the early and long-term spatial learning ability of neonates, plus the close association of FGR with the dysfunction of the central nervous system, leading to the abnormal behavior of FGR kids $(3,4)$. Hippocampus, as a part of the limbic system, plays an important role in learning and memory ability (5). VD is characterized by the function to regulate the immune system and protect the central nervous system, and it is believed that the deficiency of VD is associated with the occurrence and development of cognitive dysfunction, and the relevant studies have revealed that VD can ameliorate the cognitive 
dysfunction (6). In this study, we established the FGR models of rats through passive smoking, and later they were fed by the regular diet containing VD every day to observe and analyze their learning and memory ability as well as the expression of NMDA receptor in hippocampus. The specific procedures are reported as follows.

\section{Materials and methods}

Experiment materials. A total of 36 healthy adult Sprague-Dawley (SD) rats weighing about $250 \mathrm{~g}$ were selected ( 24 female and 12 male rats). Experiment apparatus and reagents: electronic scales (Jiangsu Hongheng Electronic Instruments Factory, Changzhou, China), electrophoresis tank (Beijing Liuyi Biotechnology Co., Ltd., Beijing, China), UV-2000 UV analyzer (Shanghai Scientific Instruments \& Materials Co., Ltd., Shanghai, China), TRIzol agent (Invitrogen, Carlsbad, CA, USA), Access RT-PCR system (Promega Corp., Madison, WI, USA), goat anti-NRI antibody (Santa Cruz Biotechnology, Inc., Santa Cruz, CA, USA), ECL kit (Santa Cruz Biotechnology, Inc.), horse anti-goat antibody (Zhongshan Golden Bridge Biotechnology Co., Ltd., Beijing, China), primer (Shanghai Boya Biotechnology Co., Ltd., Shanghai, China) and cigarettes.

Establishing the FGR animal models. We placed 24 SD female rats with 12 SD male rats (2:1) in a cage overnight, and the vaginal smear of the female rats was collected on the 2 nd day and observed under the light microscope (BX-42; Olympus, Tokyo, Japan) to identify the existence of sperm. If sperm was found on the vaginal smear of rats, the corresponding female rats were identified and placed in separate cages. Those rats were randomly divided into two groups, i.e. the normal group $(n=12)$ and the model group $(n=12)$. From the 2 nd to 21st day, the pregnant rats in the model group were placed into the smoking house 3 times for passive smoking (50 min/ time, and each time 4 cigarettes), respectively at 9:00 a.m., 12:00 a.m. and 4:00 p.m. No interventions were taken for rats in the normal group. The offspring of rats in the normal group were taken as the control group and with the 10th percentile of offspring in the control group being the standard, and the offspring of rats in the model group which were lower than the standard were selected as the observation group (7). The rats in the observation group were fed with regular diet containing VD $(0.2 \mu \mathrm{g} /(\mathrm{kg} / \mathrm{day})$ every day and the rats in the control group were only fed with regular diet every day. This study was approved by the Animal Ethics Committee of Xi'an Jiaotong University.

Determination of the serum level of $25(\mathrm{OH}) \mathrm{D}_{3}$. A total of $2 \mathrm{ml}$ fasting venous blood was withdrawn from 30 rats of each group in the morning on the 1st, 7th, 14th and 30th day after birth, and placed for $30 \mathrm{~min}$ at room temperature. After the blood samples were centrifuged at 2,750 x $\mathrm{g}$ for for $20 \mathrm{~min}$, the serum was separated and placed into the EP tube for preservation at $-70^{\circ} \mathrm{C}$. The serum level of $25-\mathrm{OH}-\mathrm{D}$ was determined using ELISA according to the $25(\mathrm{OH}) \mathrm{D}_{3}$ kit (R\&D Systems, Inc., Minneapolis, MN, USA): i) the standard sample was diluted and added; ii) incubation: the plate was blocked using membrane and placed at $37^{\circ} \mathrm{C}$ in an incubator for $30 \mathrm{~min}$ for incubation; iii) rinsing and adding the enzyme: the plate was rinsed using scrubbing solution 5 times every $20 \mathrm{sec}$, and $50 \mu \mathrm{l}$ enzyme-labeled agent was added into each well; iv) re-incubation and rinsing: the plate was blocked and placed at $37^{\circ} \mathrm{C}$ in an incubator for $30 \mathrm{~min}$ followed by rinsing 5 times; v) color development and termination: $50 \mu 1$ color-developing agent $\mathrm{A}$ and $\mathrm{B}$ was added, mixed well with the sample and placed in an incubator for incubation at $37^{\circ} \mathrm{C}$ for $15 \mathrm{~min}$ without light and then $50 \mu \mathrm{l}$ of stop buffer was added to terminate the reaction and vi) measurement: $15 \mathrm{~min}$ after the stop buffer was added, the $\mathrm{OD}_{450}$ values were measured sequentially for each well and the corresponding concentration of samples was calculated.

Morris water maze test. A self-made round pool $(50 \mathrm{~cm}$ of diameter) was prepared, to which the water mixed with ink $\left(25^{\circ} \mathrm{C}\right)$ was added. The pool was divided into 4 quadrants, and a refuge platform $(6 \times 6 \mathrm{~cm})$ was set at $1 \mathrm{~cm}$ under the water in one of the quadrants and the position was fixed. A total of 30 rats aged 30 days were respectively selected from the two groups for swimming training. Then, the rat was put into the water in one of the quadrants randomly except the one with refuge platform, and the location where the rat was put into the water was used in the following experiments, and the time from the rat being put into the water to climbing onto the refuge platform was recorded, and the rat had $15 \mathrm{sec}$ to climb onto the refuge platform. The rat which failed to climb onto the refuge platform in $120 \mathrm{sec}$ after it was put into the water was taken out of the water and put onto the refuge platform for $15 \mathrm{sec}$ of rest, and the next round of training was initiated in $120 \mathrm{sec}$, and the score of this round was $120 \mathrm{sec}$. All rats were trained for 4 times/day for 5 consecutive days. The positions of all facilities were required to be fixed and the average time that the rat needed for climbing onto the refuge platform was taken as the search latency of that day.

Passive avoidance test. A self-made transparent box $(30 \times 30 \times 40 \mathrm{~cm})$ was prepared, in which the steel tubes were fixated ( $2 \mathrm{~cm}$ in diameter) in parallel with the distance between every two tubes being $1 \mathrm{~cm}$. Electric shock machine was installed on either end of each tube, and the insulated and safe platform $(8 \times 2.5 \times 2.5 \mathrm{~cm})$ was placed on the tubes in the corner at the bottom of the transparent box. A total of 30 rats aged 30 days that had already experienced the Morris water maze test were, respectively, selected from the two groups. At the beginning of test, the rats were put onto the safe platform. Once they left the platform and touched the tubes, they would be immediately shocked. To avoid the shock, rats could jump to the safe platform, during which they might get shocked several times. The criterion for judging whether the rats learned how to avoid electric shock was set as follow: The rats could stay on the platform persistently for at least $2 \mathrm{~min}$. We recorded the number of electric shocks that the rats experienced in achieving the standard. This test was carried out for 4 days, and the average in any day was taken as the score of that day.

Measuring the brain weight. Rats $(\mathrm{n}=8)$ were selected respectively from the two groups on the 1st, 7th, 14th, 21st and 30th day, and sacrificed. The brain tissues above foramen 


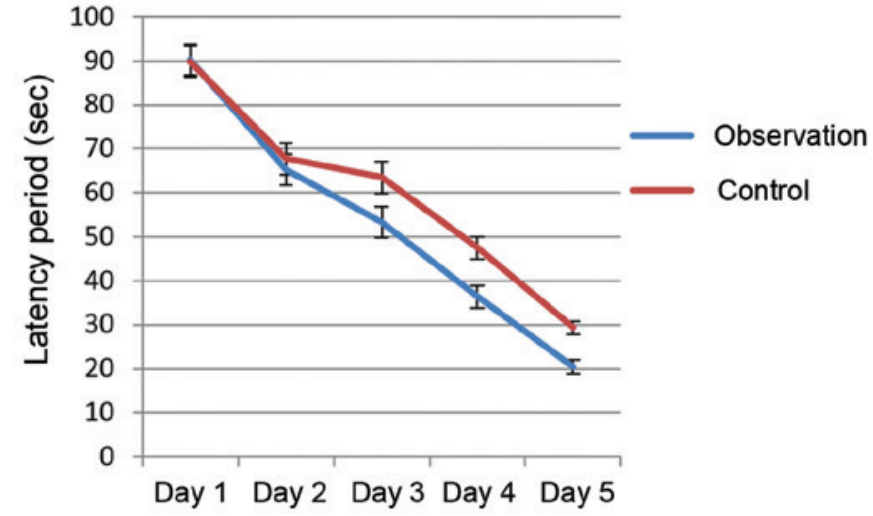

Figure 1. Comparison of the results of the Morris water maze test between the two groups at different times.

Table I. Comparison of the levels of $25(\mathrm{OH}) \mathrm{D}_{3}$ between the two groups of rats at different days (nmol/l).

\begin{tabular}{lccccc}
\hline Groups & Cases & 1 day & 7 days & 15 days & 30 days \\
\hline Observation & 30 & $53.89 \pm 3.63$ & $75.13 \pm 4.34$ & $79.23 \pm 3.24$ & $84.63 \pm 3.56$ \\
Control & 30 & $54.34 \pm 3.67$ & $56.79 \pm 4.45$ & $63.72 \pm 3.23$ & $64.52 \pm 3.73$ \\
t-test & & 0.477 & 16.160 & 18.569 & 21.362 \\
P-value & & 0.634 & $<0.0001$ & $<0.0001$ & $<0.0001$ \\
\hline
\end{tabular}

magnum were immediately separated and the wet weight of brain tissues was measured using electronic scale (being corrected to $0.001 \mathrm{~g}$ ).

Detecting the NMDA receptor in hippocampus. Rats $(\mathrm{n}=6)$ aged 30 days that had experienced both of the Morris water maze and passive avoidance test were randomly selected for determination of the mRNA expression of NRI subunit in hippocampus using RT-qPCR: a) hippocampus was isolated and preserved at $-80^{\circ} \mathrm{C}$; b) tissue RNA was extracted using TRIzol according to manufacturer's instructions; c) the total hippocampus RNA was amplified into the target fragments of DNA using Access RT-PCR system and d) PCR products were stained using ethidium bromide and $2 \%$ agarose gel electrophoresis was performed, and the bands were observed using UV-2000 UV analyzer and quantitative analysis was performed accordingly using HMIAS-2000, in which the relative expression of NRI subunit was expressed by the gray intensity ratio of NRI to GAPDH.

The protein expression of NRI subunit in hippocampus was assayed using western blotting: a) the total protein was extracted from hippocampus using the fragment method of synapse membrane and preserved at $-80^{\circ} \mathrm{C}$; b) $60 \mu \mathrm{g}$ total protein was added into the same volume of buffer and boiled for $5 \mathrm{~min}$ followed by protein separation using SDS-PAGE; c) the protein was transferred onto the nitrocellulose (NC) membrane and d) NC membrane was immersed in the blocking buffer and incubated for $30 \mathrm{~min}$ at $20^{\circ} \mathrm{C}$ to block the membrane, followed by washing the blot 3 times $(30 \mathrm{~min} /$ time), and goat anti-NRI antibody was added to the blot, followed by HRP-labeled horse anti-goat antibody. The

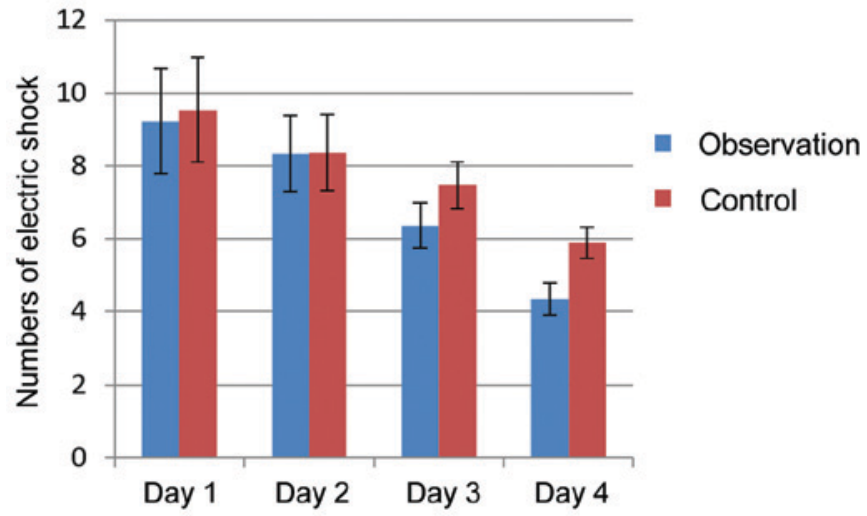

Figure 2. Comparison of the results of the passive avoidance test between the two groups at different times.

protein expression of NRI subunit was detected using ECL kit.

Statistical analysis. SPSS 19.0 software (Chicago, IL, USA) was used for processing the data. Measurement data were presented as mean $\pm \mathrm{SD}$, and t-test was performed for comparison. $\mathrm{P}<0.05$ indicated that the difference was statistically significant.

\section{Results}

Comparison of the levels of $25(\mathrm{OH}) \mathrm{D}_{3}$ between the two groups of rats on different days. No statistically significant difference was identified when comparing the levels of $25(\mathrm{OH}) \mathrm{D}_{3}$ of rats aged 1 day between the two groups ( $\mathrm{P}>0.05)$, but the levels of $25(\mathrm{OH}) \mathrm{D}_{3}$ of rats aged 7,15 and 30 days, respectively in the observation group were significantly higher than those in the control group $(\mathrm{P}<0.05$, Table I).

Comparison of the results of Morris water maze test at different times between the two groups. A shortened trend was identified in the latency of each group after the rats were trained. In the first 2 days, the latency periods of the observation and control groups were $(90.26 \pm 3.15,65.36 \pm 3.27) \mathrm{sec}$ and $(89.78 \pm 2.34,67.76 \pm 3.24)$ sec respectively, with no statistically significant difference $(\mathrm{P}>0.05)$; while on the $3 \mathrm{rd}, 4$ th and 5 th day the latency periods in the observation group, $(53.37 \pm 3.57,36.35 \pm 2.38$ and $20.45 \pm 2.47) \mathrm{sec}$, respectively, were significantly shorter than those in the control group, $(63.48 \pm 3.21,47.49 \pm 2.36$ and $29.35 \pm 2.48) \mathrm{sec}$, respectively, and the differences were statistically significant $(\mathrm{P}<0.05$, Fig. 1).

Comparison of the results of passive avoidance test between the two groups. With the training being carried out, the number of electric shocks that the rats from the two groups experienced was gradually decreased. In the first 2 days, the numbers of electric shock of the observation and control groups were $(9.23 \pm 1.45,8.34 \pm 1.26)$ and $(9.54 \pm 1.36,8.36 \pm 1.25)$, respectively, with no statistically significant difference $(\mathrm{P}>0.05)$; while in the 3rd and 4th day the numbers of electric shock in the observation group $(6.37 \pm 0.67,4.35 \pm 0.48)$, respectively, were significantly less than those in the control group (7.48 \pm 1.14 , 
Table II. Comparison of the weight of rat brain at different days between the two groups (g).

\begin{tabular}{lccccc}
\hline Groups & 1 day & 7 days & 14 days & 21 days & 30 days \\
\hline Observation & $0.178 \pm 0.013$ & $0.396 \pm 0.034$ & $0.794 \pm 0.065$ & $1.336 \pm 0.073$ & $1.398 \pm 0.085$ \\
Control & $0.212 \pm 0.027$ & $0.715 \pm 0.056$ & $1.142 \pm 0.084$ & $1.352 \pm 0.067$ & $1.412 \pm 0.092$ \\
t-test & 3.209 & 13.772 & 9.267 & 0.457 & 0.316 \\
P-value & 0.006 & $<0.0001$ & $<0.0001$ & 0.654 & 0.756 \\
\hline
\end{tabular}

Table III. Comparison of the expression of NMDA receptor in hippocampus of rats in both groups.

\begin{tabular}{lccc}
\hline Groups & $\begin{array}{c}\text { Cases } \\
(\mathrm{n})\end{array}$ & $\begin{array}{c}\text { Relative mRNA } \\
\text { expression of } \\
\text { NRI subunit }\end{array}$ & $\begin{array}{c}\text { Relative protein } \\
\text { expression of } \\
\text { NRI subunit }\end{array}$ \\
\hline Observation & 6 & $0.895 \pm 0.093$ & $0.672 \pm 0.056$ \\
Control & 6 & $0.568 \pm 0.077$ & $0.535 \pm 0.043$ \\
t-test & & 2.577 & 2.671 \\
P-value & & 0.027 & 0.023 \\
\hline
\end{tabular}

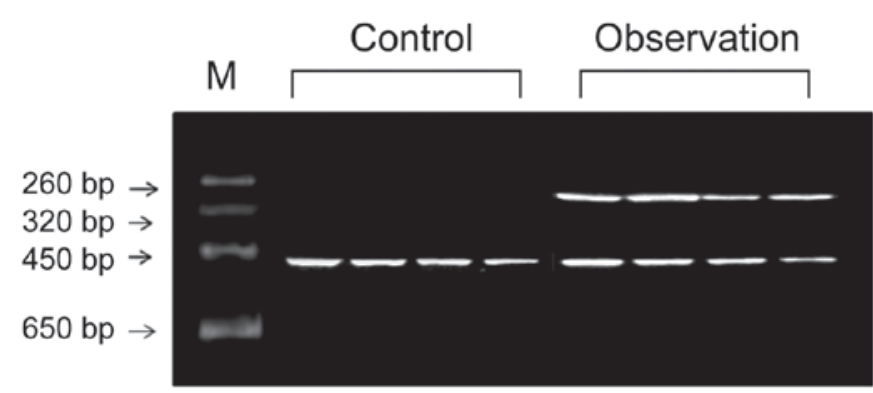

Figure 3. Analysis of RT-PCR products of mRNA of NRI subunit in rats aged 30 days (agarose gel after electrophoresis). Lane M, molecular standards; lanes 2-5, control group; lanes 6-9, observation group.

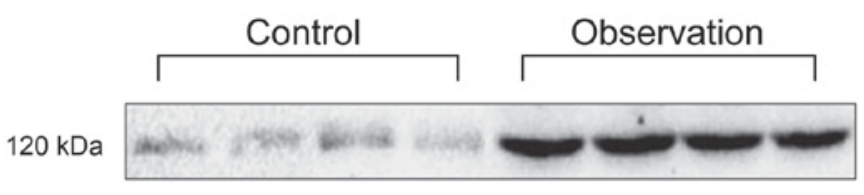

Figure 4. The western blotting result of NRI subunit of NMDA receptor in rats aged 30 days.

$5.89 \pm 0.46)$, respectively, and the differences were statistically significant ( $\mathrm{P}<0.05$, Fig. 2).

Comparison of the brain weight of rats of different age between the two groups. The brain weights of rats aged 1, 7 and 14 days in the observation group were significantly lower than those in the control group $(\mathrm{P}<0.05)$, but no obvious difference was found in the comparison of the brain weights of rats aged 21 and 30 days between the two groups ( $\mathrm{P}>0.05$, Table II).

The relative expression of mRNA and protein of NRI subunit of NMDA receptor in hippocampus of rats aged 30 days in the observation group was significantly higher than that in the control group $(\mathrm{P}<0.05$, Table III). The results of agarose gel electrophoresis and western blotting for RT-PCR product are shown in Figs. 3 and 4.

\section{Discussion}

Cognitive functions include the memory, perception, thought and imagination, and the damage to one or more of these functions can induce the dysfunction in learning and memory (8). Research has identified FGR as one of the independent risk factors for neural function deficits (9) not only for its short-term influence on the infants, but also for its long-term influence on some disease in the adult period (10).

Correlation between vitamin $D$ and cognitive functions. VD can exert an important regulatory effect in various physiological processes. Many studies have confirmed that VD can protect the nerves in the brain tissues (11). VD, after being absorbed into blood, can be transformed into the $25(\mathrm{OH})$ $\mathrm{D}_{3}$ with a high bioactivity and a nearly 3-week biological half-life, which, thus, has been applied as a general index for the assessment of VD (12). Instead of directly affecting the target organ, VD usually exerts its effect through binding with its receptor (VDR) (13). According to previous studies, the proliferation, growth and differentiation of cells in hippocampus usually begin from the 20th day of embryonic development, during which the VDR is expressed in the hippocampus, and the $25(\mathrm{OH}) \mathrm{D}_{3}$ can affect the proliferation and differentiation of cells in hippocampus, and thus the cognitive functions, indicating that VD may influence the proliferation, regulation and differentiation of neurons of the fetus (14). Studies (15-17) have identified the existence of VD metabolic enzyme and VDR in the central nervous system, suggesting that VD participates in the cognitive process (15). VD can affect the levels of neurotrophin and multiple neurotransmitters, protect the normal transduction of nerves and benefit the 
development of cognitive functions (16). Relevant studies have shown that the diameter of axon of sensory nerve in the VD therapy after nerve injuries is gradually increased, indicating that VD can promote the development of neural function and growth of nerves to a certain degree (17). In this study, VD was continuously supplemented to the FGR rats, and the level of $25(\mathrm{OH}) \mathrm{D}_{3}$ in the rats aged 7 days of the observation group was significantly higher than that in the control group and VD showed the capability to improve the cognitive functions.

Effect of VD on the brain tissues as well as the learning and memory ability of FGR rat. The results of this study revealed that the brain weights of rats on the 1st, 7th and 14th day in the observation group were obviously lower than those in the control group $(\mathrm{P}<0.05)$, which might be caused by the restricted growth and development of neurons; but the brain weights of FGR rats aged 21 and 30 days were similar to those of the normal rats $(\mathrm{P}>0.05)$, suggesting that with the time of VD supplementation being extended, the development of brain tissues has effectively gained due to the continuous structural variations and growth of neurons in hippocampus. In behavioral neuroscience, the Morris water maze test and passive avoidance test are the most common tools for the studies on the learning and memory ability (18). In this study, we found that in the Morris water maze test for the rats in both groups, there was no significant difference in comparison with the latency in the first 2 days $(\mathrm{P}>0.05)$, but in the subsequent 3 days, the latency of the observation group was shortened compared to that of the control group. Similar results were also obtained in the passive avoidance test: FGR rats, only after experiencing fewer electric shocks, could stay in the refuge platform longer than 2 min, showing that the memory ability of rats in the observation group was superior to that of the control group. Those two results of the different tests revealed that after 30 days of VD supplementation, the cognitive function of FGR rats was effectively perfected, and their learning and memory ability was also improved.

Effect of vitamin $D$ on the receptor of hippocampus. As a component of the limbic system of the brain, hippocampus not only is the region involved in the formation of learning and memory ability, but also contains abundant receptors of NMDA (19). In the development of the central nervous system, NMDA receptor participates in the survival and migration of neurons and the establishment of synaptic connection. Therefore, NMDA receptor plays an important role in maintaining the regular growth and survival of neurons in the development, and the long-term potentiation (LTP) of hippocampus, an NMDA receptor-dependent mechanism, constitutes the neural foundation for the formation of learning and memory ability (20). In this study, we found that the mRNA and protein relative expression of NRI subunit of the NMDA receptor in hippocampus of FGR rats aged 30 days was significantly higher than that in the control group $(\mathrm{P}<0.05)$. Despite the fact that the regulation mechanism on the expression of NMDA receptor remains unclear, we believe that continuous supplementation of VD can stimulate the receptor, which causes more channels to open, and, consequently, internal flow of $\mathrm{Ca}^{2+}$ is increased. Therefore, the duration of EPSP mediated by the receptor is prolonged, making the brain tissue development more susceptible to excitation, which benefits the development of neurons, and, as a result, the learning and memory ability is improved.

In conclusion, the correlation between the VD and cognitive function has been confirmed by many studies, but its mechanism remains unclear. In this study, using the FGR animal models, we confirmed that VD can improve the learning and memory ability of FGR rats, which can serve as a theoretical reference for the education of FGR children. However, the correlation between VD and cognitive development should be further identified through future relevant studies on human beings.

\section{Acknowledgements}

This study was supported by the Basic Research of Natural Science of Shaanxi Province (no. 2014JQ2-8047) and the Fundamental Research Funds for the Central Universities (no. XJJ2014072).

\section{References}

1. Baschat AA: Fetal growth restriction - from observation to intervention. J Perinat Med 38: 239-246, 2010.

2. Zeitlin J, El AM, Jarreau PH, Draper ES, Blondel B, Kunzel W, Cuttini M, Kaminski M, Gortner L, van Reempts P, et al: Impact of fetal growth restriction on mortality and morbidity in a very preterm birth cohort. J Pediatr 157: 733-739, 2010.

3. Brouillet S, Murthi P, Hoffmann P, Salomon A, Sergent F, de Mazancourt P, Dakouane-Giudicelli M, Dieudonné MN, Rozenberg P, Vaiman D, et al: EG-VEGF controls placental growth and survival in normal and pathological pregnancies: Case of fetal growth restriction (FGR). Cell Mol Life Sci 70: 511-525, 2013.

4. Baschat AA: Neurodevelopment after fetal growth restriction. Fetal Diagn Ther 36: 136-142, 2014.

5. Eichenbaum H: Time cells in the hippocampus: A new dimension for mapping memories. Nat Rev Neurosci 15: 732-744, 2014.

6. Etgen T, Sander D, Bickel H, Sander K and Förstl H: Vitamin D deficiency, cognitive impairment and dementia: A systematic review and meta-analysis. Dement Geriatr Cogn Disord 33: 297-305, 2012.

7. Stephens AS, Bentley JP, Taylor LK and Arbuckle SM: Diagnosis of fetal growth restriction in perinatal deaths using brain to liver weight ratios. Pathology 47: 51-57, 2015.

8. de Jager CA, Oulhaj A, Jacoby R, Refsum H and Smith AD: Cognitive and clinical outcomes of homocysteine-lowering B-vitamin treatment in mild cognitive impairment: A randomized controlled trial. Int J Geriatr Psychiatry 27: 592-600, 2012.

9. Kurjak A, Talic A, Honemeyer U, Stanojevic M and Zalud I: Comparison between antenatal neurodevelopmental test and fetal Doppler in the assessment of fetal well being. J Perinat Med 41: 107-114, 2012.

10. Crispi F, Bijnens B, Figueras F, Bartrons J, Eixarch E, Le Noble F, Ahmed A and Gratacós E: Fetal growth restriction results in remodeled and less efficient hearts in children. Circulation 121: 2427-2436, 2010.

11. Salari M, Janghorbani M, Etemadifar M, Dehghani A, Razmjoo H and Naderian $G$ : Effects of vitamin D on retinal nerve fiber layer in vitamin $\mathrm{D}$ deficient patients with optic neuritis: Preliminary findings of a randomized, placebo-controlled trial. J Res Med Sci 20: 372-378, 2015.

12. Mangge H, Zelzer S, Meinitzer A, Stelzer I, Schnedl WJ, Weghuber D, Fuchs D, Postolache TT, Aigner E, Datz C, et al: $25 \mathrm{OH}-\mathrm{Vitamin} \mathrm{D} 3$ levels in obesity and metabolic syndrome - Unaltered in young and not correlated to carotid IMT in all ages. Curr Pharm Des 21: 2243-2249, 2015.

13. Speeckaert MM, Speeckaert R, van Geel N and Delanghe JR: Vitamin D binding protein: A multifunctional protein of clinical importance. Adv Clin Chem 63: 1-57, 2014.

14. Harms LR, Cowin G, Eyles DW, Kurniawan ND, McGrath JJ and Burne TH: Neuroanatomy and psychomimetic-induced locomotion in C57BL/6J and 129/X1SvJ mice exposed to developmental vitamin D deficiency. Behav Brain Res 230: 125-131, 2012. 
15. Nassar MF, Amin DA, Hamed AI, Nassar JF, Abou-Zeid AE and Attaby MA: Vitamin D status and scholastic achievement in middle age childhood. J Egypt Soc Parasitol 42: 349-358, 2012.

16. Llewellyn DJ, Lang IA, Langa KM and Melzer D: Vitamin D and cognitive impairment in the elderly U.S. population. J Gerontol Bio Sci Med Sci 66: 59-65, 2011.

17. Brondum-Jacobsen P, Nordestgaard BG, Schnohr P and Benn M: 25-Hydroxyvitamin D and symptomatic ischemic stroke: An original study and meta-analysis. Ann Neurol 73: 38-47, 2013

18. Lee B, Sur BJ, Han JJ, Shim I, Her S, Lee HJ and Hahm DH: Krill phosphatidylserine improves learning and memory in Morris water maze in aged rats. Prog Neuropsychopharmacol Biol Psychiatry 34: 1085-1093, 2010.
19. Khan SA, Keaser ML, Meiller TF and Seminowicz DA: Altered structure and function in the hippocampus and medial prefrontal cortex in patients with burning mouth syndrome. Pain 155 : 1472-1480, 2014.

20. Grienberger C, Chen $X$ and Konnerth A: NMDA Receptor-dependent multidendrite $\mathrm{Ca}^{2+}$ spikes required for hippocampal burst firing in vivo. Neuron 81: 1274-1281, 2014. 